\title{
The Effects of Promotional Content on the Nutritional Quality of Children's Breakfast Cereals From Australia, New Zealand, The United Kingdom, Canada and The United States ${ }^{+}$
}

\author{
Lynne Chepulis ${ }^{1, *}$, Nadine Everson ${ }^{2}$, Jason H.Y. Wu ${ }^{3}$ and Gael Mearns ${ }^{4}$ \\ 1 Waikato Medical Research Centre, University of Waikato, Hamilton 3216, New Zealand \\ 2 Department of General Medicine, Lakes District Health Board, Rotorua 3010, New Zealand; \\ nadine.everson@lakesdhb.govt.nz \\ 3 George Institute for Global Health, University of New South Wales, Sydney 2042, Australia; \\ jwu1@georgeinstitute.org.au \\ 4 School of Clinical Sciences, Auckland University of Technology, Auckland 0627, New Zealand; \\ gael.mearns@aut.ac.nz \\ * correspondence: lynnec@waikato.ac.nz \\ † Presented at the 2018 Nutrition Society of New Zealand Annual Conference, Auckland, New Zealand, \\ 28-30 November 2018.
}

Published: 5 March 2019

Background: Breakfast is considered to be an essential meal for children, offering valuable nutrition for growing bodies. However, the nutritional value of pre-packaged children's cereals has been questioned recently, particularly as reduced nutritional quality can contribute to childhood obesity.

Methods: Nutritional composition data was collected in store and/or online from all available children's breakfast cereals during November-December 2017 from New Zealand, Australia, Canada, the UK and the USA. Cereals were included if they used promotional characters, fonts, games and bite-size pieces, or were located in the children's cereals on online shopping websites.

Results: A total of 636 products were evaluated for overall package size. Median package size was smaller in the UK when promotional characters were present and largest overall in Canada compared to other countries. After removal of duplicate products of differing package size, 487 products were analysed for nutritional composition. New Zealand and Australian products had the lowest median fat content (all $p<0.01$ ) whilst US cereals contained significantly more sugar per serve than all other countries (10.0 g vs. $7.7-9.0 \mathrm{~g}, p<0.0001)$. More than $97 \%$ of all products across all countries contained added sugars, with the average sugar content exceeding $25 \%$ in most products. The use of promotional material made no difference to the nutritional quality of children's cereals in Australia, New Zealand and the US, though UK products with characters were shown to contain more fat and carbohydrate and less protein than products without characters (all $p<0.05$ ). In Canada, products with promotional characters contained significantly more sugar ( $30.0 \mathrm{vs.} 22.8 \mathrm{~g} ; p=0.0007)$.

Conclusions: There appear to be few differences between counties with regard to the nutritional quality of children's breakfast cereals. Most products contained high quantities of sugar and a poor nutrient profile, irrespective of whether promotional materials were used.

(C) 2019 by the authors. Licensee MDPI, Basel, Switzerland. This article is an open access article distributed under the terms and conditions of the Creative Commons Attribution (CC BY) license (http://creativecommons.org/licenses/by/4.0/). 